\title{
PHYTOCHEMICAL STUDY OF ENDEMIC COSTA RICAN ANNONACEAE SPECIES Annona pittieri AND Cymbopetalum costaricense
}

\author{
JONATHAN PARRA ${ }^{1}$, CHRISTIAN DE FORD ${ }^{2}$ AND RENATO MURILLO ${ }^{3 *}$ \\ ${ }^{I}$ Pharmacy Faculty and CIPRONA, University of Costa Rica, Costa Rica. \\ ${ }^{2}$ Institute for Pharmaceutical Sciences, University of Freiburg, Germany. \\ ${ }^{3}$ Chemistry School and CIPRONA, University of Costa Rica, Costa Rica.
}

\begin{abstract}
Phytochemical profile of the Central American rainforest endemic Annonaceae species, Annona pittieri and Cymbonopetallum costaricense, were studied in search of novel bioactive compounds. The acetogenin squamocin (1) isolated from A. pittieri showed cytotoxic activity against human acute lymphocytic leukaemia with low activity against healthy blood cells. In addition, other eight compounds were isolated from A. pittieri, including a novel 2-azaanthraquinone alkaloid, 4-methoxybenzo[g]isoquinoline-5,10-dione (2). Furthermore, from C. costaricense three compounds were isolated including a novel 1-azaanthraquinone alkaloid, 6-hydroxy-9-methoxy-cleistopholine (3).
\end{abstract}

Keywords: Alkaloids, Annonaceae, Annona pittieri, Cymbopetalum costaricense, squamocin.

\section{INTRODUCTION}

Natural products are an important source of structural scaffolds for drug discovery, with particular relevance in the development of chemotherapeutics ${ }^{1}$. In particular, alkaloids and acetogenins from Annonacea species have been widely studied as potential cytotoxic compounds ${ }^{2,3}$.

The Annonaceae species, Annona pittieri Donn. Sm. and Cymbopetalum costaricense Donn. Sm. are native plants of the Costa Rican and Panamanian rainforest ${ }^{4,5}$ that are still understudied in terms of their phytochemical profile. The use of $C$. costaricense as a medicinal plant in the treatment of snake bites by Ngäbe people of Panama and Costa Rica was reported previously ${ }^{6}$, while there are no records of medicinal uses for A. pittieri. Although cyanogenic compounds were previously reported in both species ${ }^{7}$, there are no preceding studies on their phytochemical composition. Thus, in this work, A. pittieri and C. costaricense were studied as a source of new bioactive compounds.

\section{EXPERIMENTAL}

\section{Plant material}

Leaves, bark and wood of Annona pittieri were collected in La Cruz, Guanacaste, Costa Rica. Leaves, bark and wood of Cymbopetalum costaricense were collected in Sarapiquí, Heredia, Costa Rica. Both specimens were identified by the botanist Luis Poveda and stored in the Juvenal Valerio herbarium of the National University of Costa Rica.

\section{Extraction}

The plant material was dried and grounded. Separately, leaves $(550 \mathrm{~g})$ and wood (4250 g) of $A$. pittieri were extracted with a mixture of methyl tert-butyl ether (MTBE) and methanol $(\mathrm{MeOH})(9: 1)$ by maceration during $48 \mathrm{~h}$. Then, the remaining plant material was alkalized with $\mathrm{NH}_{3}(1 \%)$ and the extraction with MTBE-MeOH (9:1) was repeated. Finally, the remaining plant material was extracted once more with MTBE-MeOH (7:3). Leaves and wood (300 g) of $C$. costaricense were extracted with the same procedure mentioned above.

\section{Compound isolation}

Each crude extract was purified by open column chromatography using silica gel (70-230 mesh, Merck $\left.{ }^{\circledR}\right)$ and a gradient system of solvents of hexane, MTBE and $\mathrm{MeOH}$ mixtures. Pure compounds were isolated from fractions using flash chromatography with silica gel $(60$ mesh, Merck®) and thin-layer chromatography (TLC) with silica gel $\left(60 \mathrm{~F}_{254}\right.$, Merck $\left.{ }^{\circledR}\right)$.

\section{Structural elucidation}

Structures of the isolated compound were elucidated by nuclear magnetic resonance spectroscopy (NMR). The NMR spectra were recorded in deuterated chloroform $\left(\mathrm{CDCl}_{3}\right)$ and deuterated methanol $\left(\mathrm{CD}_{3} \mathrm{OD}\right)$ on a Bruker ${ }^{\circledR}$ Ascend $\AA$ $600 \mathrm{MHz}$ and a Varian ${ }^{\circledR}$ Mercury ${ }^{\circledR} 400 \mathrm{MHz}$ spectrometers. The structure 1 was confirmed by mass spectrometry (MS) on a high-resolution atmospheric pressure chemical ionization (HRAPCI) with an Orbitrap ${ }^{\circledR}$ mass spectrometer (Thermo Fisher $\left.{ }^{\circledR}\right)$, while the structure 2 on electrospray ionization (ESI) with Quadrupole Time-of-flight tandem (QTOF) mass spectrometer (Waters ${ }^{\circledR}$ ), and the structure 3 on a direct infusion electrospray ionization (DIESI) with Triple Quadrupole Linear Ion Traps (QTRAP) mass spectrometer (SCIEX®).

\section{Cellular assays}

Cytotoxicity of squamocin isolated from A. pittieri was tested against three tumour cell lines: CCRF-CEM (human T-cell acute lymphoblastic leukaemia), CEM-ADR5000 (human T-cell acute lymphoblastic leukaemia resistant to doxorubicin) and MIA-PaCa-2 (human pancreatic carcinoma), as well as against peripheral blood mononuclear cells (PBMC) from healthy human subjects; according to the method published by Calderón $\mathrm{et}^{\mathrm{al}} \mathrm{l}^{8}$. PBMCs were isolated from human buffy coats obtained from the Freiburg University Clinic, Freiburg, Germany (ethical permission number from the ethics commission, University of Freiburg: $356 / 13$; 2013). Briefly, the cells were maintained at $37{ }^{\circ} \mathrm{C}$ under $5 \%$ $\mathrm{CO}_{2}$ in RPMI 1640 medium supplemented with $10 \%$ heat-inactivated fetal bovine serum, $100 \mathrm{mg} / \mathrm{mL}$ streptomycin, and $100 \mathrm{U} / \mathrm{mL}$ penicillin. The cells were seeded in 96-well plates $\left(1.2 \times 10^{4}\right.$ cells/well for MIA-PaCa- 2 cells, $4 \times 10^{4}$ cells/well for leukemic cells, and $2 \times 10^{5} \mathrm{PBMCs} /$ well in $150 \mu \mathrm{L}$ complete medium). Squamocin was dissolved in DMSO, and the cells were incubated for $24 \mathrm{~h}$ with various concentrations of squamocin or the positive control, respectively. Camptothecin and doxorubicin were used as the positive controls, and DMSO $0.1 \%$ was the solvent control. The viability of the tumour cells was quantified using an MTT assay. The $\mathrm{IC}_{50}$ values were obtained by nonlinear regression using the GraphPad ${ }^{\circledR} \operatorname{Prism}{ }^{\circledR}$ 5. The data are expressed as means \pm SD.

Squamocin (1): White wax. ${ }^{1} \mathrm{H}$ NMR $\left(\mathrm{CDCl}_{3}, 600 \mathrm{MHz}, J / \mathrm{Hz}\right): \delta 2.24(2 \mathrm{H}, \mathrm{d}$ $J=7.8, \mathrm{H}-3), 1.52$ (2H, m, H-4), 1.23 (m, H-5, 6, 7, 8, 9, 10, 11, 12, 30, 31), 1.41 (m, H-13, 27, 29), 1.36 (m, H-14), 3.38 (1H, m, H-15), 3.82 (1H, m, H-16), 1.56 $\left(3 \mathrm{H}, \mathrm{m}, \mathrm{H}_{\mathrm{a}}-17,18,21\right), 1.95\left(3 \mathrm{H}, \mathrm{m}, \mathrm{H}_{\mathrm{b}}-17,18,21\right), 3.90(1 \mathrm{H}, \mathrm{m}, \mathrm{H}-19), 3.82$ $(1 \mathrm{H}, \mathrm{m}, \mathrm{H}-20), 1.80\left(1 \mathrm{H}, \mathrm{d}, J=6, \mathrm{H}_{\mathrm{a}}-22\right), 1.89\left(1 \mathrm{H}, \mathrm{d}, J=6, \mathrm{H}_{\mathrm{b}}-22\right), 3.94(1 \mathrm{H}, \mathrm{m}$, H-23), 3.88 (1H, m, H-24), 1.35 (m, H-25), 1-37 (m, $\left.\mathrm{H}_{\mathrm{a}}-26\right), 1.65$ (1H, d, J=9.6, 
$\left.\mathrm{H}_{\mathrm{b}}-26\right), 3.57$ (1H, m, H-28), 1.23 (m, H-32, 33), 0.86 (3H, t, $\left.J=6.6, \mathrm{H}-34\right), 6.98$ $(1 \mathrm{H}, \mathrm{d}, J=1.2, \mathrm{H}-35), 4.98$ (1H, dq, $J=1.2,6.6, \mathrm{H}-36), 1.39$ (3H, d, $J=6.6, \mathrm{H}-37)$;

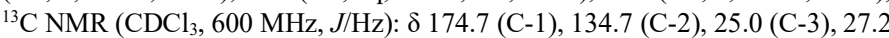
(C-4), 29.0-29,6 (C-5, 6, 7, 8, 9, 10, 11, 12, 30, 31), 25.5 (C-13), 32.2 (C-14), 74.4 (C-15), 83.5 (C-16), 28.7 (C-17, 18, 21), 82.7 (C-19), 82.3 (C-20), 24.6 (C22), 83.0 (C-23), 71.4 (C-24), 33.0 (C-25), 21.8 (C-26), 37.3 (C-27), 71.8 (C28), 37.1 (C-29), 31.7 (C-32), 22.4 (C-33), 13.8 (C-34), 149.5 (C-35), 77.6 (C36), 19.0 (C-37). HRAPCI (negative mode) $\mathrm{m} / \mathrm{z} 621.4736[\mathrm{M}-\mathrm{H}]^{-}$(calcd for $\left.\mathrm{C}_{37} \mathrm{H}_{65} \mathrm{O}_{7}^{-}, 621.4730\right)$. TLC Rf $0.42\left(\mathrm{CHCl}_{3}-\mathrm{MeOH} ; 95: 5\right)$.

5-Methoxyflavone: Pale yellow needles. ${ }^{1} \mathrm{H}$ NMR $\left(\mathrm{CDCl}_{3}, 600 \mathrm{MHz}, \mathrm{J} / \mathrm{Hz}\right)$ $\delta 6.75(1 \mathrm{H}, \mathrm{s}, \mathrm{H}-3), 6.82(1 \mathrm{H}, \mathrm{d}, J=8.4, \mathrm{H}-6), 7.58(1 \mathrm{H}, \mathrm{dd}, J=8.4, \mathrm{H}-7), 7.14$ (1H, d, J=8.4, H-8), 7.90 (2H, m, H-2', 6'), 7.51 (2H, m, H-3', 5'), $7.50(1 \mathrm{H}, \mathrm{s}$, $\mathrm{H}-4$ '), $3.99\left(3 \mathrm{H}, \mathrm{s}, \mathrm{H}-5-\mathrm{OCH}_{3}\right) ;{ }^{13} \mathrm{C} \mathrm{NMR}\left(\mathrm{CDCl}_{3}, 600 \mathrm{MHz}, \mathrm{J} / \mathrm{Hz}\right): \delta 161.3(\mathrm{C}-$ 2), 109.2 (C-3), 178.6 (C-4), 159.8 (C-5), 106.5 (C-6), 133.9 (C-7), 110.3 (C-8), 158.4 (C-9), 114.7 (C-10), 131.5 (C-1'), 126.2 (C-2', 6'), 129.1 (C-3', 5'), 131.5 (C-4'), 56.6 (C-5-OCH $)$. TLC Rf $0.81\left(\mathrm{C}_{6} \mathrm{H}_{6}-\mathrm{CH}_{2} \mathrm{Cl}_{2}-\mathrm{MTBE} ; 1: 1: 1\right)$

5,2'-Dimethoxyflavone: Pale yellow needles. ${ }^{1} \mathrm{H}$ NMR $\left(\mathrm{CDCl}_{3}, 600 \mathrm{MHz}\right.$, $J / \mathrm{Hz}): \delta 7.09(1 \mathrm{H}, \mathrm{s}, \mathrm{H}-3), 6.82(1 \mathrm{H}, \mathrm{d}, J=7.8, \mathrm{H}-6), 7.56(1 \mathrm{H}, \mathrm{dd}, J=7.8, \mathrm{H}-7)$, $7.11(1 \mathrm{H}, \mathrm{d}, J=7.8, \mathrm{H}-8), 7.04\left(1 \mathrm{H}, \mathrm{d}, J=8.4, \mathrm{H}-3^{\prime}\right), 7.47(1 \mathrm{H}, \mathrm{dd}, J=8.4,7.2, \mathrm{H}-$ 4'), $7.11\left(1 \mathrm{H}, \mathrm{m}, \mathrm{H}-5\right.$ '), $7.90\left(1 \mathrm{H}, \mathrm{d}, J=7.2, \mathrm{H}-6\right.$ '), $4.01\left(3 \mathrm{H}, \mathrm{s}, \mathrm{H}-5-\mathrm{OCH}_{3}\right), 3.94$ $\left(3 \mathrm{H}, \mathrm{s}, \mathrm{H}-2{ }^{\prime}-\mathrm{OCH}_{3}\right) ;{ }^{13} \mathrm{C} \mathrm{NMR}\left(\mathrm{CDCl}_{3}, 600 \mathrm{MHz}, J / \mathrm{Hz}\right): \delta 158.7(\mathrm{C}-2), 114.3$ (C-3), 179.3 (C-4), 159.8 (C-5), 106.2 (C-6), 133.7 (C-7), 110.3 (C-8), 158.6 (C9), 114.3 (C-10), 120.5 (C-1'), 158.2 (C-2'), 111.8 (C-3'), 132.4 (C-4'), 120.8 (C-5'), $129.2\left(\mathrm{C}-6\right.$ '), $56.6\left(\mathrm{C}-5-\mathrm{OCH}_{3}\right), 55.8\left(\mathrm{C}-2^{\prime}-\mathrm{OCH}_{3}\right)$. TLC Rf $0.71\left(\mathrm{C}_{6} \mathrm{H}_{6-}\right.$ $\mathrm{CH}_{2} \mathrm{Cl}_{2}$-MTBE; 1:1:1).

4-Methoxybenzo[g|isoquinoline-5,10-dione (2): Yellow solid. ${ }^{1} \mathrm{H}$ NMR and ${ }^{13} \mathrm{C}$ NMR data, see Table 1. ESIQTOF (positive mode) $\mathrm{m} / \mathrm{z} 240.066[\mathrm{M}+\mathrm{H}]^{+}$ (calcd for $\left.\mathrm{C}_{14} \mathrm{H}_{10} \mathrm{NO}_{3}{ }^{+}, 240.0661\right)$. TLC Rf $0.44\left(\mathrm{CHCl}_{3}-\mathrm{MeOH} ; 6: 4\right)$.

Liriodenine: Yellow solid. ${ }^{1} \mathrm{H}$ NMR $\left(\mathrm{CDCl}_{3}, 600 \mathrm{MHz}, J / \mathrm{Hz}\right): \delta 7.15(1 \mathrm{H}, \mathrm{d}$, $J=4.8, \mathrm{H}-3), 7.75(1 \mathrm{H}, \mathrm{dd}, J=4.8, \mathrm{H}-4), 8.73(1 \mathrm{H}, \mathrm{dd}, J=4.8, \mathrm{H}-5), 8.45(1 \mathrm{H}, \mathrm{dd}$, $J=3,7.8, \mathrm{H}-8), 7.51(1 \mathrm{H}$, ddd, $J=3,7.8,7.5, \mathrm{H}-9) 7.70(1 \mathrm{H}, \mathrm{ddd}, J=3,7.8,7.5, \mathrm{H}-$ $10), 8.58(1 \mathrm{H}, \mathrm{dd}, J=3,7.8, \mathrm{H}-11), 6.32\left(2 \mathrm{H}, \mathrm{d}, J=3, \mathrm{H}-1-\mathrm{OCH}_{2} \mathrm{O}-2\right) ;{ }^{13} \mathrm{C}$ NMR $\left(\mathrm{CDCl}_{3}, 600 \mathrm{MHz}, \mathrm{J} / \mathrm{Hz}\right): \delta 148.4(\mathrm{C}-1), 152.1$ (C-2), 103.3 (C-3), 136.1 (C-3a), 123.6 (C-3b), 124.7 (C-4), 144.4 (C-5), 144.9 (C-6a), 182.6 (C-7), 131.0 (C-7a), 128.6 (C-8), 128.7 (C-9), 134.2 (C-10), 127.5 (C-11), 133.0 (C-11a), 107.9 (C$11 \mathrm{~b}), 102.7\left(\mathrm{C}-1-\mathrm{OCH}_{2} \mathrm{O}-2\right)$. TLC Rf $0.71\left(\mathrm{CHCl}_{3}-i \mathrm{PrOH} ; 95: 5\right)$.

Tamgermanetin: Yellow solid. ${ }^{1} \mathrm{H}$ NMR $(\mathrm{MeOD}, 600 \mathrm{MHz}, J / \mathrm{Hz}): \delta 7.11$ $(1 \mathrm{H}, \mathrm{d}, J=1.8, \mathrm{H}-2), 6.79$ (1H, d, $J=8.4, \mathrm{H}-5), 7.02(1 \mathrm{H}, \mathrm{dd}, J=1.8,8.4, \mathrm{H}-6), 7.42$ (1H, d, $J=15.6, \mathrm{H}-7), 6.40$ (1H, d, $J=15.6, \mathrm{H}-8), 7.05$ (2H, d, $J=8.4, \mathrm{H}-2$ ', 6'), $6.71\left(2 \mathrm{H}, \mathrm{d}, J=8.4, \mathrm{H}-3^{\prime}, 5^{\prime}\right), 2.75$ ( $\left.2 \mathrm{H}, \mathrm{t}, J=7.2, \mathrm{H}-7^{\prime}\right), 3.46$ ( $\left.2 \mathrm{H}, \mathrm{t}, J=7.2, \mathrm{H}-8^{\prime}\right)$, $3.87\left(3 \mathrm{H}, \mathrm{s}, \mathrm{H}-4-\mathrm{OCH}_{3}\right) ;{ }^{13} \mathrm{C} \mathrm{NMR}(\mathrm{MeOD}, 600 \mathrm{MHz}, J / \mathrm{Hz}): \delta 128.3(\mathrm{C}-1)$, 111.5 (C-2), 149.8 (C-3), 149.3 (C-4), 116.3 (C-5), 123.2 (C-6), 142.0 (C-7), 118.7 (C-8), 169.2 (9), 130.7 (C-1'), 130.7 (C-2', 6'), 116.3 (C-3', 5'), 156.9 (C4'), 35.8 (C-7'), 42.5 (C-8'), $56.4\left(\mathrm{C}^{\prime}-4-\mathrm{OCH}_{3}\right)$. TLC Rf $0.38\left(\mathrm{CHCl}_{3}-i \mathrm{PrOH}\right.$; 9:1)

(+)-Catechin: Brown solid. ${ }^{1} \mathrm{H}$ NMR (MeOD, $\left.600 \mathrm{MHz}, \mathrm{J} / \mathrm{Hz}\right): \delta 4.56(1 \mathrm{H}, \mathrm{d}$, $J=7.8, \mathrm{H}-2), 3.97(1 \mathrm{H}$, ddd, $J=5.4,8.4,7.8, \mathrm{H}-3), 2.50\left(1 \mathrm{H}, \mathrm{dd}, J=16.2,8.4, \mathrm{H}_{\mathrm{a}-}\right.$ 4), $2.84\left(1 \mathrm{H}, \mathrm{dd}, J=16.2,8.4, \mathrm{H}_{\mathrm{b}}-4\right), 5.93(1 \mathrm{H}, \mathrm{s}, \mathrm{H}-6), 5.85(1 \mathrm{H}, \mathrm{s}, \mathrm{H}-8), 6.83$ (1H, d, J=1.8, H-2'), 6.76 (1H, d, $\left.J=8.4, \mathrm{H}-5^{\prime}\right), 6.71$ (1H, d, $J=1.8,8.4$, H-6'); ${ }^{13} \mathrm{C}$ NMR (MeOD, $\left.600 \mathrm{MHz}, J / \mathrm{Hz}\right): \delta 82.7$ (C-2), 68.7 (C-3), 28.4 (C-4), 100.8 (C-4a), 157.4 (C-5), 96.3 (C-6), 157.6 (C-7), 95.5 (C-8), 156.8 (C-8a), 132.1 (C1'), 115.2 (C-2'), 146.1 (C-3'), 146.2 (C-4'), 116.1 (C-5'), 120.0 (C-6'). TLC Rf $0.18\left(\mathrm{CHCl}_{3}-i \mathrm{PrOH} ; 9: 1\right)$

( \pm )-Marmesin: Brown solid. ${ }^{1} \mathrm{H}$ NMR $\left(\mathrm{CDCl}_{3}, 600 \mathrm{MHz}, J / \mathrm{Hz}\right): \delta 6.22(1 \mathrm{H}$, d, $J=9.3, \mathrm{H}-3), 7.59(1 \mathrm{H}, \mathrm{d}, J=9.3, \mathrm{H}-4), 7.22(1 \mathrm{H}, \mathrm{s}, \mathrm{H}-5), 6.74(1 \mathrm{H}, \mathrm{s}, \mathrm{H}-8)$, $4.74\left(1 \mathrm{H}, \mathrm{t}, J=8.4, \mathrm{H}-2^{\prime}\right), 3.21$ (2H, m, H-3'), 1.24 (3H, s, H-4'- $\left.\mathrm{CH}_{3 \mathrm{a}}\right), 1.37$ (3H, s, H-4'- $\left.\mathrm{CH}_{3 \mathrm{~b}}\right) ;{ }^{13} \mathrm{C} \mathrm{NMR}\left(\mathrm{CDCl}_{3}, 600 \mathrm{MHz}, \mathrm{J} / \mathrm{Hz}\right): \delta 161.9(\mathrm{C}-2), 112.5(\mathrm{C}-3)$, 143.8 (C-4), 112.5 (C-4a), 123.6 (C-5), 125.2 (C-6), 163.3 (C-7), 98.1 (C-8), 155.8 (C-8a), 91.2 (C-2'), 29.6 (C-3'), 71.8 (C-4'), $24.4\left(\mathrm{C}_{\mathrm{a}}-4^{\prime}-\mathrm{CH}_{3}\right), 26.3\left(\mathrm{C}_{\mathrm{b}^{-}}\right.$ 4'- $\left.\mathrm{CH}_{3}\right)$. TLC Rf $0.38\left(\mathrm{C}_{6} \mathrm{H}_{6}-\mathrm{CH}_{2} \mathrm{Cl}_{2}-\mathrm{MTBE} ; 4: 4: 2\right)$.

Methyl ent-16a,17-dihydroxy-kauran-19-oate: Yellow solid. ${ }^{1} \mathrm{H}$ NMR $\left(\mathrm{CDCl}_{3}, 600 \mathrm{MHz}, \mathrm{J} / \mathrm{Hz}\right): \delta 0.77\left(1 \mathrm{H}, \mathrm{m}, \mathrm{H}_{\mathrm{ax}}-1\right), 1.81\left(1 \mathrm{H}, \mathrm{m}, \mathrm{H}_{\mathrm{eq}}-1\right), 1.42(1 \mathrm{H}$, $\left.\mathrm{m}, \mathrm{H}_{\mathrm{ax}}-2\right), 1.82\left(1 \mathrm{H}, \mathrm{m}, \mathrm{H}_{\mathrm{eq}}-2\right), 0.99\left(1 \mathrm{H}, \mathrm{m}, \mathrm{H}_{\mathrm{ax}}-3\right), 2.16\left(1 \mathrm{H}, \mathrm{m}, \mathrm{H}_{\mathrm{eq}}-3\right), 1.02$ $(1 \mathrm{H}, \mathrm{dd}, J=1.8,12, \mathrm{H}-5), 1.73\left(1 \mathrm{H}, \mathrm{m}, \mathrm{H}_{\mathrm{ax}}-6\right), 1.83\left(1 \mathrm{H}, \mathrm{m}, \mathrm{H}_{\mathrm{eq}}-6\right), 1.43(1 \mathrm{H}, \mathrm{m}$,
$\left.\mathrm{H}_{\mathrm{ax}}-7\right), 1.63\left(1 \mathrm{H}, \mathrm{m}, \mathrm{H}_{\mathrm{eq}}-7\right), 0.98(1 \mathrm{H}, \mathrm{m}, \mathrm{H}-9), 1.50\left(1 \mathrm{H}, \mathrm{m}, \mathrm{H}_{\mathrm{ax}}-11\right), 1.59(1 \mathrm{H}$, $\left.\mathrm{m}, \mathrm{H}_{\mathrm{eq}}-11\right), 1.49\left(1 \mathrm{H}, \mathrm{m}, \mathrm{H}_{\mathrm{ax}}-12\right), 1.57\left(1 \mathrm{H}, \mathrm{m}, \mathrm{H}_{\mathrm{eq}}-12\right), 2.03(1 \mathrm{H}, \mathrm{m}, \mathrm{H}-13), 1.60$ $\left(1 \mathrm{H}, \mathrm{m}, \mathrm{H}_{\mathrm{ax}}-14\right), 1.92\left(1 \mathrm{H}, \mathrm{m}, \mathrm{H}_{\mathrm{eq}}-14\right), 1.43\left(1 \mathrm{H}, \mathrm{m}, \mathrm{H}_{\mathrm{a}}-15\right), 1.56\left(1 \mathrm{H}, \mathrm{m}, \mathrm{H}_{\mathrm{b}}-15\right)$, $3.66\left(1 \mathrm{H}, \mathrm{d}, J=11.1, \mathrm{H}_{\mathrm{a}}-17\right), 3.76\left(1 \mathrm{H}, \mathrm{d}, J=11.1, \mathrm{H}_{\mathrm{b}}-17\right), 1.16(3 \mathrm{H}, \mathrm{s}, \mathrm{H}-18), 0.82$ $(3 \mathrm{H}, \mathrm{s}, \mathrm{H}-20), 3.64\left(3 \mathrm{H}, \mathrm{s}, \mathrm{H}-19-\mathrm{OCH}_{3}\right) ;{ }^{13} \mathrm{C}$ NMR $\left(\mathrm{CDCl}_{3}, 600 \mathrm{MHz}, J / \mathrm{Hz}\right): \delta$ 40.6 (C-1), 18.9 (C-2), 38.0 (C-3), 43.7 (C-4), 56.9 (C-5), 21.9 (C-6), 41.9 (C7), 44.6 (C-8), 55.7 (C-9), 39.4 (C-10), 18.3 (C-11), 26.0 (C-12), 45.2. (C-13), 37.1 (C-14), 53.1 (C-15), 82.1 (C-16), 66.4 (C-17), 28.6 (C-18), 178.8 (C-19), 15.1 (C-20), $51.2\left(\mathrm{C}-19-\mathrm{OCH}_{3}\right)$. TLC Rf $0.27\left(\mathrm{C}_{6} \mathrm{H}_{6}-\mathrm{CH}_{2} \mathrm{Cl}_{2}-\mathrm{MTBE} ; 4: 4: 2\right)$.

4-Methyl-2(1H)-quinolinone: Yellow solid. ${ }^{1} \mathrm{H}$ NMR $\left(\mathrm{CDCl}_{3}, 400 \mathrm{MHz}\right.$, $J / \mathrm{Hz}): \delta 6.69(1 \mathrm{H}, \mathrm{d}, J=1.2, \mathrm{H}-3), 8.24(1 \mathrm{H}, \mathrm{dd}, J=1.2,7.6, \mathrm{H}-5), 7.8(1 \mathrm{H}, \mathrm{ddd}$, $J=1.2,7.6,7.6, \mathrm{H}-6), 7.87$ (1H, ddd, $J=1.2,7.6,7.6, \mathrm{H}-7), 8.19$ (1H, dd, $J=1.2$, 7.6, $\mathrm{H}-8), 2.71\left(4 \mathrm{H}, \mathrm{d}, J=1.2, \mathrm{H}-4-\mathrm{CH}_{3}\right), 9.70(\mathrm{H}-\mathrm{NH}) ;{ }^{13} \mathrm{C} \mathrm{NMR}\left(\mathrm{CDCl}_{3}, 400\right.$ $\mathrm{MHz}, J / \mathrm{Hz}): \delta 161.0(\mathrm{C}-2), 128.0(\mathrm{C}-3), 152.8$ (C-4), 116.5 (C-4a), 128.2 (C-5), 134.2 (C-6), 136.3 (C-7), 127.1 (C-8), 143.4 (C-8a), $22.6\left(\mathrm{C}-4-\mathrm{CH}_{3}\right)$. TLC Rf $0.76\left(\mathrm{CHCl}_{3}-i \mathrm{PrOH} ; 9: 1\right)$.

6,7-Dimethoxy-1-methyl-2(1H)-quinolinone: Orange amorphous solid. ${ }^{1} \mathrm{H}$ $\mathrm{NMR}\left(\mathrm{CDCl}_{3}, 400 \mathrm{MHz}, J / \mathrm{Hz}\right): \delta 6.40(1 \mathrm{H}, \mathrm{d}, J=7.2, \mathrm{H}-3), 6.99(1 \mathrm{H}, \mathrm{d}, J=7.2$, $\mathrm{H}-4), 7.80(1 \mathrm{H}, \mathrm{s}, \mathrm{H}-5), 6.85(1 \mathrm{H}, \mathrm{s}, \mathrm{H}-8), 3.59\left(3 \mathrm{H}, \mathrm{s}, \mathrm{H}-\mathrm{NCH}_{3}\right), 4.00(3 \mathrm{H}, \mathrm{s}, \mathrm{H}-$ 6- $\left.-\mathrm{OCH}_{3}\right), 3.97\left(3 \mathrm{H}, \mathrm{s}, \mathrm{H}-7-\mathrm{OCH}_{3}\right) ;{ }^{13} \mathrm{C} \mathrm{NMR}\left(\mathrm{CDCl}_{3}, 400 \mathrm{MHz}, \mathrm{J} / \mathrm{Hz}\right): \delta 162.6$ (C-2), 105.8 (C-3), 131.5 (C-4), 120.5 (C-4a), 107.8 (C-5), 149.8 (C-6), 153.8 (C-7), $106.2(\mathrm{C}-8), 133.0(\mathrm{C}-8 \mathrm{a}), 37.0\left(\mathrm{C}-\mathrm{NCH}_{3}\right), 56.0\left(\mathrm{C}-6-\mathrm{OCH}_{3}\right), 56.2(\mathrm{C}-7-$ $\left.\mathrm{OCH}_{3}\right)$. TLC Rf $0.65\left(\mathrm{CHCl}_{3}-i \mathrm{PrOH} ; 9: 1\right)$.

6-Hydroxy-9-methoxy-cleistopholine (3): Purple needles. ${ }^{1} \mathrm{H}$ NMR and ${ }^{13} \mathrm{C}$ NMR data, see Table 2. ESIQTRAP (positive mode) m/z 269.21 [M] $]^{+}$TLC Rf $0.47\left(\mathrm{CHCl}_{3}-i \mathrm{PrOH} ; 9: 1\right)$.

\section{RESULTS AND DISCUSSION}

In this work, it is reported the phytochemical profile of two native Annonaceae species from Costa Rican rainforest. Furthermore, the cytotoxic activity of an acetogenin is described, and two novel alkaloids are reported.

The acetogenin squamocin $(\mathbf{1})^{9}$; the flavonoids 5 -methoxyflavone ${ }^{10}, 5,2^{\prime}$ dimethoxyflavone ${ }^{11}$, and catechin $^{12}$; the aporphine alkaloid liriodenine ${ }^{13}$; the tyramine tamgermanetin ${ }^{14}$; the coumarin marmesin ${ }^{15}$; and ent-kaurane diterpene methyl ent-16 $\alpha, 17$-dihydroxy-kauran-19-oate ${ }^{16}$ were isolated from Annona pittieri and identified by NMR.
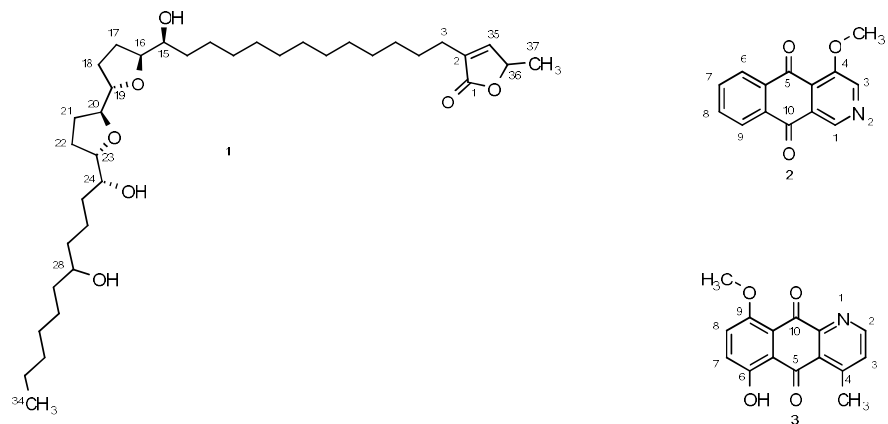

Figure 1. Compounds isolated from Annona pittieri (1 and 2) and Cymbopetalum costaricense (3).

Acetogenins compounds have been widely studied as cytotoxic agents, which activity is explained through inhibition of mitochondrial complex I (NADH:ubiquinone oxidoreductase) of the respiratory chain and inhibition of the sodium-potassium ATPase ${ }^{17}$. Squamocin (1) isolated from A. pittieri leaves showed activity against pancreatic carcinoma and leukaemia cells (Figure 2), while its higher activity was against human T-cell acute lymphoblastic leukaemia resistant to doxorubicin (CEM-ADR500). Furthermore, the cytotoxic activity was lower against healthy blood cells (Figure 3), showing selective activity against cancer cells. Although cytotoxic activity in leukaemia cells was reported previously for squamocin ${ }^{18}$. This results demonstrated in particular, a higher activity against resistant cell lines. A similar cytotoxic profile was described for other acetogenins and cell lines ${ }^{19,20}$. 


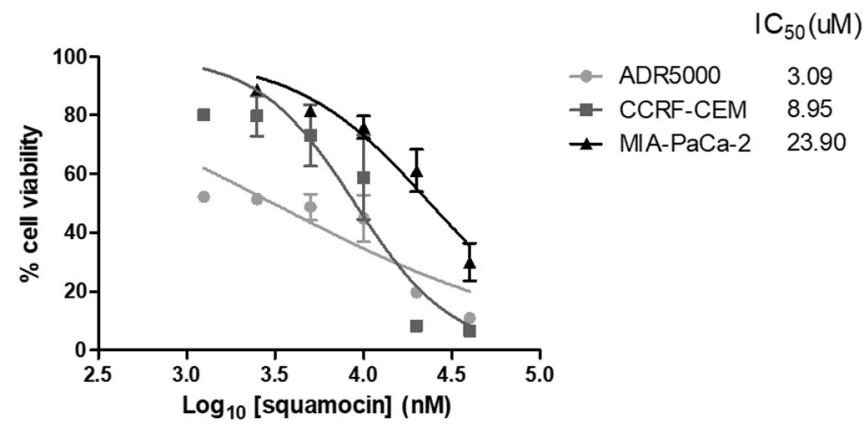

Figure 2. Cell viability of the $24 \mathrm{~h}$ treatment with squamocin isolated from $A$. pittieri in cancer cell lines. Results presented mean $\pm \mathrm{SD}(\mathrm{n}=3)$.

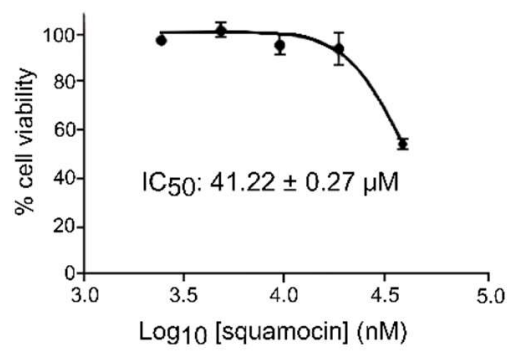

Figure 3. Cell viability of the $24 \mathrm{~h}$ treatment with squamocin isolated from $A$. pittieri PBMC cells. Results represent mean $\pm \mathrm{SD}(\mathrm{n}=3)$.

In addition, a novel 2-azaanthraquinone alkaloid, 4-methoxybenzo[ $g]$ isoquinoline5,10-dione (2), was isolated from A. pittieri stem. The ESI-TOF MS of 2 in positive mode showed a molecular ion at $m / z 240.066[\mathrm{M}+\mathrm{H}]^{+}$, suggesting a molecular formula of $\mathrm{C}_{14} \mathrm{H}_{9} \mathrm{NO}_{3}$. The NMR data (Table 1) showed signals characteristics for aromatic protons at $\delta_{\mathrm{H}} 8.29,7.88(2 \mathrm{H})$ and 8.43 , with a correlation between them in the H-H COSY spectrum. Additionally, the signals at $\delta_{\mathrm{H}} 8.29$ and 8.43 correlated with $\delta_{\mathrm{C}} 181.9$ and 180.9 , respectively in the HMBC spectrum, suggesting a benzoquinone-like system. A signal at $\delta_{\mathrm{H}} 9.18$, which showed a correlation in H-H COSY with a signal at $\delta_{\mathrm{H}} 7.63$, is typical of alpha protons to nitrogen in the isoquinoline-like system, which agrees with a 2 azaanthracene system. Furthermore, a signal at $\delta_{\mathrm{C}} 167.9$ showed correlations with $\delta_{\mathrm{H}} 7.63$ and 4.09 , which is characteristic of methoxyl groups, suggesting the structure 2.

Table 1. ${ }^{1} \mathrm{H}$ RMN (600 MHz) y ${ }^{13} \mathrm{C}$ RMN $(600 \mathrm{MHz})$ data for 2. $\mathrm{CDCl}_{3}, \delta$ [ppm] (J [Hz]).

\begin{tabular}{|c|c|c|}
\hline Position & $\boldsymbol{\delta}_{\mathbf{H}}$ & $\boldsymbol{\delta}_{\mathbf{C}}$ \\
\hline 1 & $9.18 \mathrm{~d}(4.2)$ & 155.2 \\
\hline 3 & $7.63 \mathrm{~d}(4.2)$ & 125.5 \\
\hline 4 & - & 167.9 \\
\hline $4 \mathrm{a}$ & - & 142.5 \\
\hline 5 & - & 181.9 \\
\hline $5 \mathrm{a}$ & - & 133.1 \\
\hline 6 & $8.29 \mathrm{dd}(1.8,7.2)$ & 127.7 \\
\hline 7 & $7.88 \mathrm{ddd}(1.8,7.2,6)$ & 135.1 \\
\hline 8 & $7.88 \mathrm{ddd}(1.8,7.2,6)$ & 135.2 \\
\hline 9 & $8.43 \mathrm{dd}(1.8,7.2)$ & 128.2 \\
\hline $9 \mathrm{a}$ & - & 132.7 \\
\hline 10 & - & 180.9 \\
\hline $10 \mathrm{a}$ & - & 149.4 \\
\hline $4-\mathrm{OCH}_{3}$ & $4.09 \mathrm{~s}$ & 53.6 \\
\hline
\end{tabular}

There are published reports of 1-azaanthraquinone alkaloid in others species of Annonaceae family, such as cleistopholine isolated from Cleistopholis patens $^{21}$ and 5-hydroxy-6-methoxycleistopholine isolated from Porcelia macrocarpa ${ }^{22}$. However, this is the first report of a 2-azaanthraquinone structure with a methoxyl group in the pyridine ring.
From Cymbopetalum costaricense, the quinolinone alkaloids, 4-methyl-2(1H)quinolinone ${ }^{23}$ and 6,7-dimethoxy-1-methyl-2(1H)-quinolinone ${ }^{24}$, were isolated and identified by NMR. Despite these compounds are known structures, this is the first report of their biosynthetic origin.

In addition, a novel 1-azaanthraquinone alkaloid, 6-hydroxy-9-methoxycleistopholine (3), was isolated from C. costaricense. The ESI-QTRAP MS of 3 in positive mode showed a molecular ion at $\mathrm{m} / z 269.21[\mathrm{M}]^{+}$, suggesting a molecular formula of $\mathrm{C}_{15} \mathrm{H}_{11} \mathrm{NO}_{4}$, which agrees with NMR data. Moreover, signals at $\mathrm{m} / z 254.02$ and $\mathrm{m} / \mathrm{z} 226.07$ suggested chemical transformations of demethylation and decarbonylation, respectively. The NMR data (Table 2) displayed characteristics signals of aromatic protons at $\delta_{\mathrm{H}} 7.42$ and 8.68 , which correlate each other in the $\mathrm{H}-\mathrm{H}$ COSY spectrum. Besides, the signal at $\delta_{\mathrm{H}} 8.68$ could be related to the alpha position to nitrogen in a pyridine ring, likewise discussed for 2. Finally, the signal at $\delta_{\mathrm{H}} 2.90$, which correlate to $\delta_{\mathrm{C}} 152.8$ in the HMBC spectrum, suggesting a methyl group substituting the pyridine scaffold.

Table 2. ${ }^{1} \mathrm{H}$ RMN (400 MHz) and ${ }^{13} \mathrm{C}$ RMN (400 MHz) data for 3. $\mathrm{CD}_{3} \mathrm{Cl}, \delta$ $[\mathrm{ppm}](\mathrm{J}[\mathrm{Hz}])$.

\begin{tabular}{|c|c|c|}
\hline Position & $\boldsymbol{\delta}_{\mathbf{H}}$ & $\boldsymbol{\delta}_{\mathbf{C}}$ \\
\hline 2 & $8.68 \mathrm{~d}(4.8)$ & 152.0 \\
\hline 3 & $7.42 \mathrm{~d}(4.8)$ & 131.1 \\
\hline 4 & - & 152.8 \\
\hline $4 \mathrm{a}$ & - & 128.6 \\
\hline 5 & - & 183.3 \\
\hline $5 \mathrm{~b}$ & - & 159.2 \\
\hline 6 & - & 169.9 \\
\hline 7 & $7.60 \mathrm{~d}(8)$ & 120.8 \\
\hline 8 & $6.83 \mathrm{~d}(8)$ & 111.8 \\
\hline 9 & - & 158.4 \\
\hline $9 \mathrm{a}$ & - & 125.7 \\
\hline 10 & - & 183.3 \\
\hline $10 \mathrm{a}$ & - & 148.2 \\
\hline $4-\mathrm{CH}_{3}$ & $2.90 \mathrm{~s}$ & 22.8 \\
\hline $9-\mathrm{OCH}_{3}$ & $3.96 \mathrm{~s}$ & 56.0 \\
\hline
\end{tabular}

Two more aromatic proton signals at $\delta_{\mathrm{H}} 6.83$ and 7.60 suggest the presence of another aromatic system, where $\delta_{\mathrm{H}} 7.60$ correlated with $\delta_{\mathrm{C}} 183.3$, a typical carbonyl signal, in the HMBC spectrum. Moreover, protons at $\delta_{\mathrm{H}} 6.83$ and 7.60 correlated with $\delta_{\mathrm{C}} 169.9$ and 158.4 , respectively, which are typical signals of phenoxyl group. Furthermore, the signal characteristic of phenoxy groups at $\delta_{\mathrm{H}}$ 6.83, correlated in the HMBC spectrum with $\delta_{\mathrm{C}}$ 158.4. All the HMBC correlations (Figure 3 ) are consistent with the structure 3.

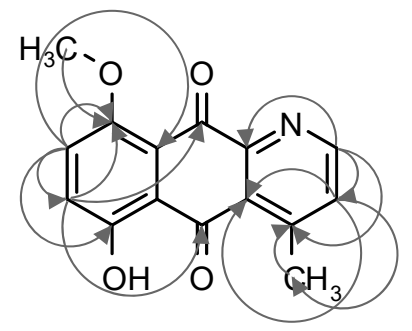

Figure 3. HMBC correlations for 3.

The structure $\mathbf{3}$ is closely related to cleistopholine, an alkaloid mentioned above, isolated from Annonaceae species, such as Cleistopholis patens ${ }^{21}$ and Annona Cherimolia ${ }^{25}$. Despite reports of hydroxyl and methoxyl derivatives of cleistopholine ${ }^{26}$, this is the first report of the 6-hydroxy-9-methoxycleistopholine structure.

\section{CONCLUSION}

Annonaceae family is a well-known source of bioactive compounds. In this work, a cytotoxic acetogenin and two novel alkaloids from Annona pittieri and Cymbopetalum costaricense were described, supporting the importance of native species from the tropical rainforest as a source of bioactive compounds. In particular, Cymbopetalum costaricense proved to be a source of alkaloids with novel structures. 


\section{ACKNOWLEDGMENT}

The authors would like to acknowledge Rodrigo Muñoz Arrieta and Randall Loaiza Montoya from the Centro Nacional de Innovaciones Biotecnológicas (CENIBiot) for the mass spectrometry determination by ESI-QTRAP. This work was supported by resources of the Vice-rectory of Research of the University of Costa Rica (project number B2028).

\section{REFERENCES}

1. Newman, D. J.; Cragg, G. M. Natural Products as Sources of New Drugs over the Nearly Four Decades from 01/1981 to 09/2019. J. Nat. Prod. 2020, 83 (3), 770-803.

2. Tundis, R.; Xiao, J.; Loizzo, M. R. Annona Species (Annonaceae): A Rich Source of Potential Antitumor Agents? Ann. N. Y. Acad. Sci. 2017, 1398 (1), 30-36.

3. Neske, A.; Ruiz Hidalgo, J.; Cabedo, N.; Cortes, D. Acetogenins from Annonaceae Family. Their Potential Biological Applications. Phytochemistry 2020, 174 (February), 112332.

4. Zamora, N.; Jiménez, Q.; Poveda, L. Árboles de Costa Rica Vol. II, 1st ed.; INBio: Santo Domingo de Heredia, 2000.

5. Daguerre, N.; Condit, R.; Perez, R. Trees of Panama and Costa Rica; Princeton University Press: New Jersey, 2010.

6. Joly, L. G.; Guerra, S.; Séptimo, R.; Solís, P. N.; Correa A., M. D.; Gupta, M. P.; Levy, S.; Sandberg, F.; Perera, P. Ethnobotanical Inventory of Medicinal Plants Used by the Guaymi Indians in Western Panama. Part II. $J$. Ethnopharmacol. 1990, 28 (2), 191-206.

7. Thomsen, K.; Brimer, L. Cyanogenic Constituents in Woody Plants in Natural Lowland Rain Forest in Costa Rica. Bot. J. Linn. Soc. 1997, 124 (3), 273-294.

8. Calderón, C.; De Ford, C.; Castro, V.; Merfort, I.; Murillo, R. Cytotoxic Clerodane Diterpenes from Zuelania Guidonia. J. Nat. Prod. 2014, 77 (3), 455-463.

9. Fujimoto, Y.; Eguchi, T.; Kakinuma, K.; Ikekawa, N.; Sahai, M.; Gupta, Y. K. Squamocin, a New Cytotoxic Bis-Tetrahydrofuran Containing Acetogenin from Annona Squamosa. Chem. Pharm. Bull. 1988, 36 (12), 4802-4806.

10. Kingsbury, C. A.; Looker, J. H. Carbon- 13 Spectra of Methoxyflavones. J. Org. Chem. 1975, 40 (8), 1120-1124.

11. Lee, S.; Moon, B.-H.; Park, Y.; Lee, E.; Hong, S.; Lim, Y. Methyl Substitution Effects on $1 \mathrm{H}$ and 13C NMR Data of Methoxyflavones. Bull. Korean Chem. Soc Soc 2008, 29 (9), 1793-1796.

12. Davis, A. L.; Cai, Y.; Davies, A. P.; Lewis, J. R. 1H And13C NMR Assignments of Some Green Tea Polyphenols. Magn. Reson. Chem. 1996, 34 (11), 887-890.

13. Zhang, Z.; ElSohly, H. N.; Jacob, M. R.; Pasco, D. S.; Walker, L. A.; Clark, A. M. New Sesquiterpenoids from the of Root of Guatteria Multivenia. $J$. Nat. Prod. 2002, 65 (6), 856-859.

14. Nawwar, M.; Swilam, N.; Hashim, A.; Al-Abd, A.; Abdel-Naim, A.; Lindequist, U. Cytotoxic Isoferulic Acidamide from Myricaria Germanica (Tamaricaceae). Plant Signal. Behav. 2013, 8 (1), e22642.

15. Joo, S.-H.; Lee, S.-C.; Kim, S.-K. UV Absorbent, Marmesin, from the Bark of Thanakha,Hesperethusa Crenulata L. J. Plant Biol. 2004, 47 (2), 163-165.

16. Etse, J. T.; Gray, A. I.; Waterman, P. G. Chemistry in the Annonaceae, Xxiv. Kaurane and Kaur- 16-Ene Diterpenes from the Stem Bark of Annona Reticulata. J. Nat. Prod. 1987, 50 (5), 979-983.

17. Bermejo, A.; Figadère, B.; Zafra-Polo, M.-C.; Barrachina, I.; Estornell, E.; Cortes, D. Acetogenins from Annonaceae: Recent Progress in Isolation, Synthesis and Mechanisms of Action. Nat. Prod. Rep. 2005, 22, 269-303.

18. Zhu, X. F.; Liu, Z. C.; Xie, B. F.; Li, Z. M.; Feng, G. K.; Xie, H. H.; Wu, S. J.; Yang, R. Z.; Wei, X. Y.; Zeng, Y. X. Involvement of Caspase-3 Activation in Squamocin-Induced Apoptosis in Leukemia Cell Line HL-60. Life Sci. 2002, 70 (11), 1259-1269.

19. Oberlies, N. H.; Croy, V. L.; Harrison, M. L.; McLaughlin, J. L. The Annonaceous Acetogenin Bullatacin Is Cytotoxic against MultidrugResistant Human Mammary Adenocarcinoma Cells. Cancer Lett. 1997, 115 (1), 73-79.

20. Oberlies, N.; Chang, C.; Mclaughlin, J. L. Structure - Activity Relationships of Diverse Annonaceous Acetogenins Against. J. Med. Chem 1997, 40 (97), 2102-2106.

21. Waterman, P. G.; Muhammad, I. Sesquiterpenes and Alkaloids from Cleistopholis Patens. Phytochemistry 1985, 24 (3), 523-527.

22. Chaves, M. H.; De Santos, L. A.; Lago, J. H. G.; Roque, N. F. Alkaloids from Porcelia Macrocarpa. J. Nat. Prod. 2001, 64 (2), 240-242.
23. Ferguson, J.; Zeng, F.; Alwis, N.; Alper, H. Synthesis of 2 ( $1 \mathrm{H}$ ) Quinolinones via Pd-Catalyzed Oxidative Cyclocarbonylation of 2 Vinylanilines. Org. Lett. 2013, 15 (6), 1998-2001.

24. Toda, J.; Sakagami, M.; Goan, Y.; Simakata, M.; Saitoh, T.; Horiguchi, Y.; Sano, T. Synthesis of Methoxy-2-Quinolones via Pummerer-Type Cyclization of N-Aryl-N-Methyl-3-(Phenylsulfinyl)Propionamides. Chem. Pharm. Bull. (Tokyo). 2000, 48 (12), 1854-1861.

25. Ríos, J. L.; Cortés, D.; Valverde, S. Acetogenins, Aporphinoids, and Azaanthraquinone from Annona Cherimolia Seeds. Planta Med. 1989, 55, 321-323.

26. Konoshima, T.; Kozuka, M.; Koyama, J.; Okatani, T.; Tagahara, K.; Tokuda, H. Studies on Inhibitors of Skin Tumor Promotion, VI. Inhibitory Effects of Quinones on Epstein-Barr Virus Activation. J. Nat. Prod. 1989, 52 (5), $987-$ 995. 